\title{
Factors Influencing Polychronous Group Sustainability as a Model of Working Memory
}

\author{
Panagiotis Ioannou ${ }^{1}$, Matthew Casey $^{2}$, and André Grüning ${ }^{1}$ \\ 1 Department of Computing, University of Surrey, Guildford, Surrey, GU2 7XH, UK \\ 2 Pervasive Intelligence Ltd, Stoneleigh, Frimley Road, Ash Vale, Surrey, GU12 5PN, UK
}

\begin{abstract}
Working memory is the system that underlies the temporary storage and manipulation of information. However, the exact biological basis and neural mechanisms of cognitive function and working memory has yet to be fully uncovered. The temporary storage of information has been correlated with enhanced spiking activity in the form of persistent reverbaration for several seconds, as shown in vivo experiments. Several computational models have been designed to help our understanding of the conditions under which persistent activity can be sustained in cortical circuits. Here, we will focus on one such model that has shown promise, that uses polychronisation and short term synaptic dynamics to achieve this reverberation, and explore it with respect to different physiological parameters in the brain, including size of the network, number of synaptic connections, small-world connectivity, maximum axonal conduction delays, and type of cells (excitatory or inhibitory). We show that excitation and axonal conduction delays greatly affect the sustainability of spatio-temporal patterns of spikes called polychronous groups, even though the large variance in some of our results suggest that further exploration is needed on a larger scale of neuronal populations.
\end{abstract}

Keywords: Spiking neural networks, polychronization, working memory

\section{Introduction}

The elevated, sustained and selective firing activity of individual neurons during working memory tasks has been observed in a number of in vivo electrophysiological experiments $[1,2,3]$. A number of models have been suggested to explain this elevated firing frequency of neurons, mainly in the form of recurrent neural networks based on NMDA receptor-mediated synaptic dynamics $[4,5,6,7,8,9,10]$. The main shortcoming of these models is that memories are represented by specific non-overlapping groups of spiking neurons. The extension of the memory content in such networks increases the overlap between the memory representations and subsequently, the activation of one representation speads to others, resulting in an uncontrolable excitation. This limitation can be overcome by a model that accounts for the precise spike-timing patterns of firing of individual neurons. In [11], memories are represented by extensively overlapping groups of neurons that exhibit stereotypical time-locked spatiotemporal spike-timing patterns, called polychronous groups (PNGs). The synapses forming such PNGs are subject to associative synaptic plasticity in the form of both long-term and short-term spike-timing dependent plasticity (STDP). While long-term potentiation is essential in PNG formation, it was shown that short-term plasticity can temporarily strengthen the synapses of selected PNGs and lead to an increase in the spontaneous reactivation rate of these PNGs, which is consistent with the in vivo recordings of individual neurons during working memory (WM) tasks. These short term changes were simulated through associative short-term synaptic plasticity via STDP, where synaptic changes - that decay to baseline within a few seconds are induced by the classical STDP protocol.

The short-term mechanism selectively affects synapses according to the relative spike timing of preand post-synaptic neurons. This differs from the short-term synaptic strengthening used in previous working memory models mentioned above, which are not associative, and hence non-selectively affect all synapses belonging to the same presynaptic neuron [11]. By selectively activating a PNG, the short term mechanism strengthens the synapses of their constituent neurons, and in conjuction with the stochastic synaptic noise, their reactivation rate increases and is persistent for a few seconds, resulting in activity similar to that observed in vivo during working memory tasks [11]. Due to the spike-timing nature of activated polychronous groups, the selective activation of a PNG does not spread in the whole network initiating uncontrollable activity. This way one can expand the sustainable memory content and maintain a large number of items, without having an extreme increase in the neuronal population. 
As brain imaging techiques have shown, during working memory tasks there is a variety of interconnected areas that are being activated. These areas exhibit striking structural, physiological and functional differences. There are many different types of neurons, with differences in size, shape and other physiological properties. Each area exhibits its own physiology and anatomy with some areas varying greatly. These differences among others include size of neurons, length of axons, density of axons, connectivity pattern, number of excitatory and inhibitory neurons. We will explore some fundamental structural differences and see what effect these differences can have on the spike timing theory of working memory and the sustainability of PNGs. In this paper we test the effect of network size, number of connections, axonal delays, small-world connectivity, and ratio of excitatory to inhibitory neurons. The work presented here focuses on how the quality of PNGs changes if various network parameters are changed and continues our work from [12] which focused on how the quantity of PNGs changed when network parameters were changed.We show that the maximum axonal conduction delays and number of excitatory neurons have a significant contribution to the PNG sustainability.

\section{Method}

We need to find a way to measure the quality of a PNG when interpreted as an item in working memory. Unfortunately, literature is not very clear on how to measure aspects of working memory [13] such as sustainability, item retention, delay interval and the decay of memory traces etc. Here we therefore measure PNG quality in two ways: 1 . by their strength, and by 2 . their duration.

We understand as the strength of a PNG the activation of the neurons that belong to the PNG for the duration of the simulation as a percentage of neurons activated over total number of neurons in a PNG, where PNG membership is determined as in [14]. Here we calculate strength as the area under curve of a PNG activation rate plot (Fig. 1). We assume that the greater the strength and activation rate of the memory trace, the greater will be the objective amount of information remembered, representing for example the amount of accurately remembered details connected to a memory item.

We define the duration of a PNG as the time interval between the onset of the stimulation where the activation frequency increases until drops back down to a baseline (Fig. 1), representing the duration of the sustained activation rate in the context of confidence on remembering the substance or general meaning of a scene or memory or just the existence of a memory trace. For us this duration represents the duration of the memory. To bring sustainability strength and duration in context, if we have big values in both strength and duration, then the memory of an item or a scene will be remembered accurately with a lot of detail (strength) and for a long time (duration). Or in another example, if you have a small sustainability strength value but with a big sustainability duration value, that could represent a memory that is remebered for a long period of time however without a lot of detail.

We follow the methodolgy used in [11], in which the original network consists of 1000 neurons, 800 excitatory (regular spiking pyramidal neurons), 200 inhibitory (GABAergic fast spiking interneurons) representing standard neocortical parameters. Excitatory synaptic connections have random delays up to $20 \mathrm{~ms}$. Inhibitory connections have $1 \mathrm{~ms}$ delays. The connectivity probability is 0.1 . We use the simple model of spiking neurons (eqs 1,2), as proposed by Izhikevich [15]. Variable $v$ represents the membrane potential of the neuron; $u$ represents the so-called recovery variable, and provides negative feedback to $v$; and $I$ represents synaptic or injected currents. The parameter $a$ describes the time scale of the recovery variable $u$. Smaller values result in slower recovery. The parameter $b$ describes the sensitivity of the recovery variable $u$ to the subthreshold fluctuations of the membrane potential $v$. The parameter $c$ describes the after-spike reset value of the membrane potential $v$. The parameter $d$ describes after-spike reset of the recovery variable $u$.

$$
\begin{aligned}
& v^{\prime}=0.04 v^{2}+5 v+140-u+I \\
& u^{\prime}=a(b v-u) \text { with reset conditions, if } v \geq+30 \mathrm{mV}, \text { then }\left\{\begin{array}{l}
v \leftarrow c \\
u \leftarrow u+d
\end{array}\right.
\end{aligned}
$$

Excitatory to inhibitory and all inhibitory connections are non-plastic. Excitatory synaptic strengths change according to the STDP rule [16]. That is, the magnitude of change of synaptic strength between a pre- and a postsynaptic neuron depends on the timing of spikes: The synapse gets potentiated if the presynaptic spike arrives at the postsynaptic neuron before the postsynaptic neuron fires; whereas the synapse gets depressed if the presynaptic spike arrives at the postsynaptic neuron after that fired. Thus, 
what matters is not the timing of spiking per se but the exact timing of the arrival of presynaptic spikes before postsynaptic targets. The magnitude of change for potentiation equals $A_{+} e^{-\frac{\Delta t}{\tau}}$; and for synaptic depression is $A_{-} e^{-\frac{\Delta t}{\tau}}$, where $\Delta t$ is the inter-spike interval between the arrival of the presynaptic spike and the postsynaptic spike, $\tau=20 \mathrm{~ms}, A_{+}=0.1$, and $A_{-}=0.12$. The synaptic strengths are bound within the interval $[0 \ldots 8] \mathrm{mV}$, which implies that the simultaneous arrival of at least three pre-synaptic spikes is needed to reliably elicit a post-synaptic response. About $10-20$ optimal pre-then-post spike pairs are needed to increase the synaptic strength of a weak synapse to the maximum value.

The efficacy of synaptic transmission for synapses connecting excitatory neurons are also scaled up or down, relative to a baseline, on a short timescale. With short-term STDP, input to neuron $i$ at time $t, I_{i}(t)$, equals $\sum_{j \in J} s_{i j}\left(1+s d_{i j}\right)$, where $s_{i j}$ is synaptic weight for the synapse between neuron $j$ and $i$; and $J$ is the set of presynaptic neurons whose spike arrived at neuron $i$ at time $t$. The effect of a presynaptic spike is scaled up or down by the factor $s d$, where this variable is different for each synapse; follows the classical STDP rule with the parameters $A_{+}, A_{-}$as above; and in the absence of synaptic activity it decays back to 0 with a time constant 5 seconds. Therefore, 1) in the absence of synaptic activity the synaptic efficacy does not change; 2 ) pre-then-post spikes temporarily increase the synaptic efficacy; and 3) post-then-pre spikes temporarily decrease the synaptic efficacy.
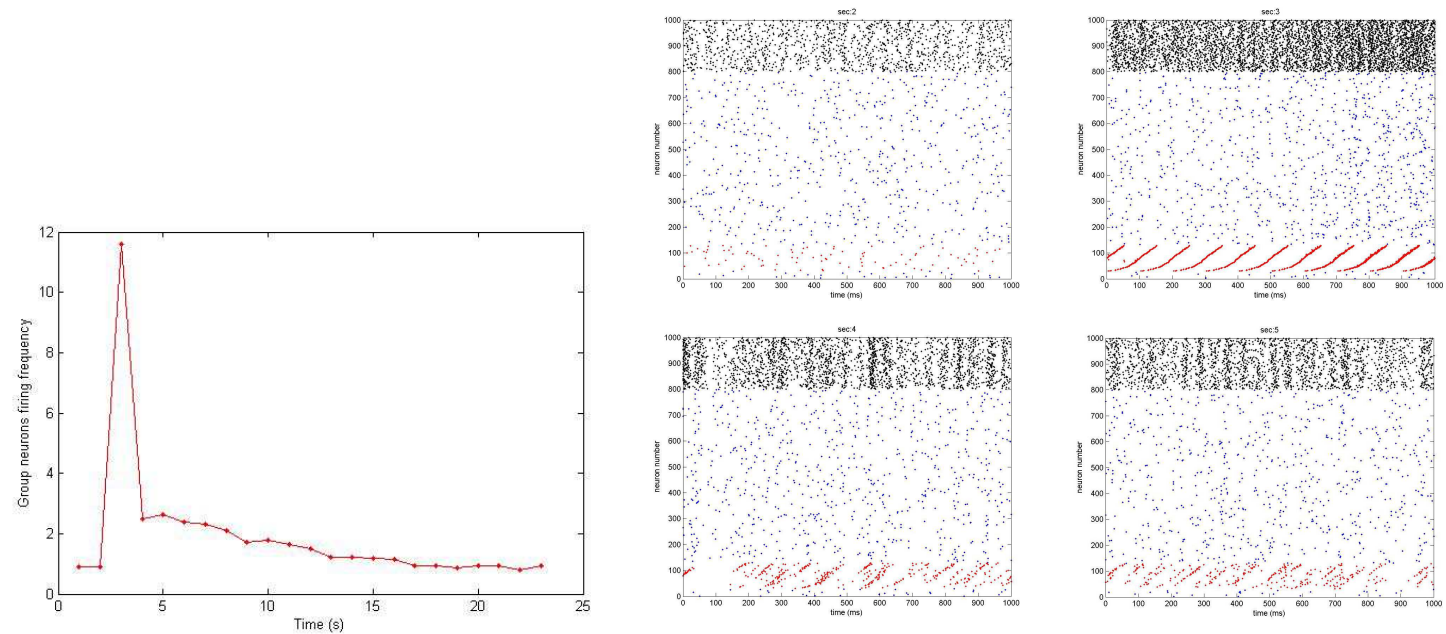

Fig. 1: Left: Firing frequency of a PNG. At the 3rd second, in which we stimulate the group 10 times, its firing frequency increases 10 fold. Then it decays back to a baseline, here 23 seconds after the stimulation. We measure sustainability in two ways: By strength, represented by the area under the curve, and duration, represented by the time the groups' firing frequency drops down to $2 \mathrm{~Hz}$. Right: Snapshots of network activity 3-6s. PNG neurons (red) are ordered according to their firing pattern for better visualisation. Blue denote excitatory and black inhibitory neurons.

Here we explore what effect some fundamental parameters have on the sustainability strength and duration of a PNG. To begin with, we run the simulations for a period of time to find emerging PNGs as described in [14]. To achieve persistent 'reverberation', we select one PNG, and we stimulate the intra-PNG neurons sequentially with the corresponding polychronous pattern at $100 \mathrm{~ms}$ intervals during a one second interval to temporarily increase the intra-PNG synaptic efficacy (see Fig. 1). To explore sustainability we run different simulations and we vary each time (while keeping everything else constant): the network size (keeping the group size percentage towards the whole network the same), the number of neuronal synaptic connections, the maximum axonal conduction delays between neurons, the connectivity pattern (small world connectivity moving from completely localized connections to completely random, assigning at the same time delays according to the corresponding synaptic distances), and then we vary the percentage of excitatory towards inhibitory neurons in the network. We run 10 simulations for each experiment to check consistency of our results. 
ICANN2014, 142, v1: 'Factors Influen...'
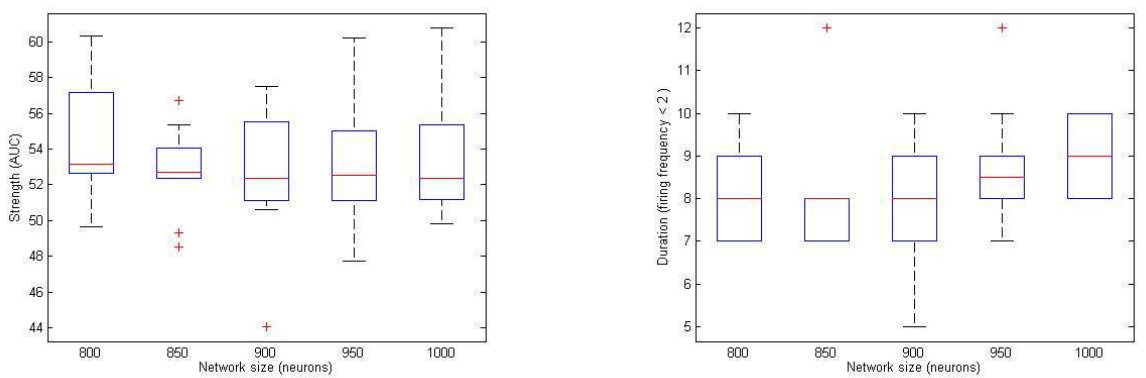

Fig. 2: Sustainability of polychronous groups of various network sizes. The network size does not affect sustainability strength, but slightly increases the duration on networks consisting of 950 and 1000 neurons respectively. Boxplots in all figures denote the median, the $25^{t h}$ and $75^{t h}$ percentiles, extreme points and outliers $(+)$.

\section{Experiments and evaluation}

\subsection{Network Size}

In the first set of experiments we will explore the decay of the memory traces with respect to different network sizes. It is important to study decay in relation to network size in order to relate actual physiological variations, including the differences in brain size between brain regions, human individuals, and different mammals. The cortex has a layered structure, with each layer having different neuronal populations, as well as neuronal variation. Other distinct brain regions have fundamental structural differences i.e. different neuronal populations [17]. This inspired us to conduct this first set of experiments, in which we explored how sustainability of a PNG is affected when we vary the network size. Here we use the model as described in the method section, however we vary the network size: 800, 850, 900, 950 and 1000 neurons, keeping the ratio excitatory to inhibitory $4: 1$. We keep the PNG size towards the rest of the network constant $(10 \%)$.

Results are as follow: we notice that the network size does not affect the sustainability of a PNG (see Fig. 2). There is a slight increase at the duration on networks consisting of 950 and 1000 neurons respectively, however there is a great variance and very small difference between the means of each set of experiments to suggest significant changes. This can also be quantified with an analysis of variance (ANOVA) resulting in a very large $p$ value for strength $(p=0.7833)$ and duration $(p=0.3563)$, indicating that the differences between column means are not significant. To relate this with short-term memory sustainability, our results suggest that networks of different sizes, or brain areas of different sizes or bigger cortices, seem to have the same memory trace sustainability. The relative insensitivity of the quality of PNGs to global size of the network can be explained because a PNG is a local motif in a network and this local motif does not change if the global size is varied. The number of PNGs, i.e. their quantity however does depend on the global size of the network [12] This could suggest that memory decay per se is not a key factor in intelligence or working memory capacity, but is a result of the ability to represent larger memory content and repertoir with larger networks [12], or due to some other executive control mechanisms [18].

\subsection{Number of Synaptic Connections}

A synapse, in addition to its ability to regulate different specific functions like STDP, has a relatively small size [17]. Thus, if cortical areas consist of billions of neurons, then the synapses are in the order of trillions. The structure for example of pyramidal cells varies considerably between cortical areas and species in terms of the size and complexity of their dendritic arborisation [19]. Moreover, it is important to study how the numbers of synapses per neuron affect memory trace sustainability, as synaptogenesis explodes synaptic numbers during early brain development, while synaptic pruning facilitates changes in neural structure by reducing the overall number of neurons and synapses, leaving more efficient synaptic configurations. In [11], a value of 100 synapses per neuron is used. Consequently, in our experiments, we varied the number of synapses from 80 to 100 in scale of 5 to explore how sustainability is affected keeping the number of neurons and all other parameters fixed.

The large variances suggest that the number of connections is not an important parameter in the quality of a PNG (see Fig. 3). This is also evident after an ANOVA showing large $p$ values for strength 

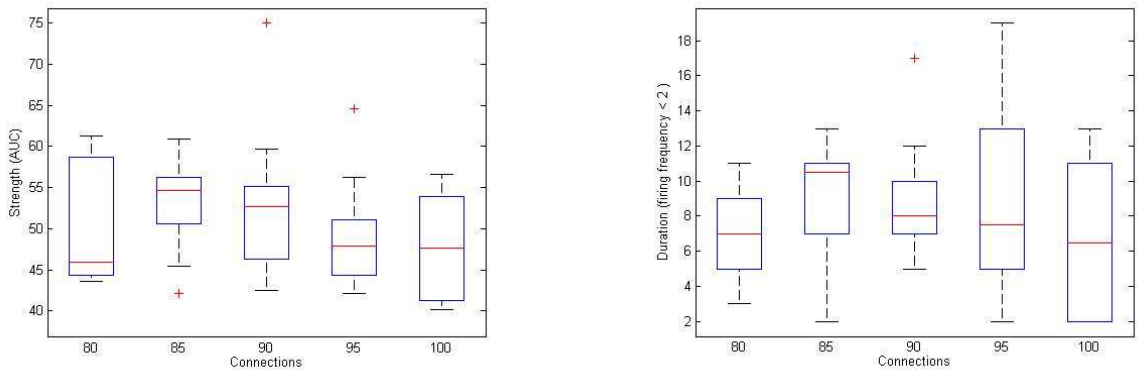

Fig. 3: Sustainability of polychronous groups when we vary the number of synaptic connections per neuron. There is an optimal mean value for strength and duration for 85 connections per neuron, increased from 80 connections per neuron and gradually decreases for 90, 95, and 100 connections per neuron.

$(p=0.3631)$ and duration $(p=0.3563)$. The relative insensitivity of the quality of PNGs to changes in the number of synapses does not influence an existing PNG as spiking in the network is sparse. That is a local PNG does not "feel" that is has more synapses, because as before most of a neurons' incomimg synapses are silent - and once the anchor and mother neurons are fired, the (sparse) additional activity through the (additional) synapses does not change group behaviour. However, synapses greatly affect the quantity of PNGs, as shown in [12].

\subsection{Range of Axonal Delays}
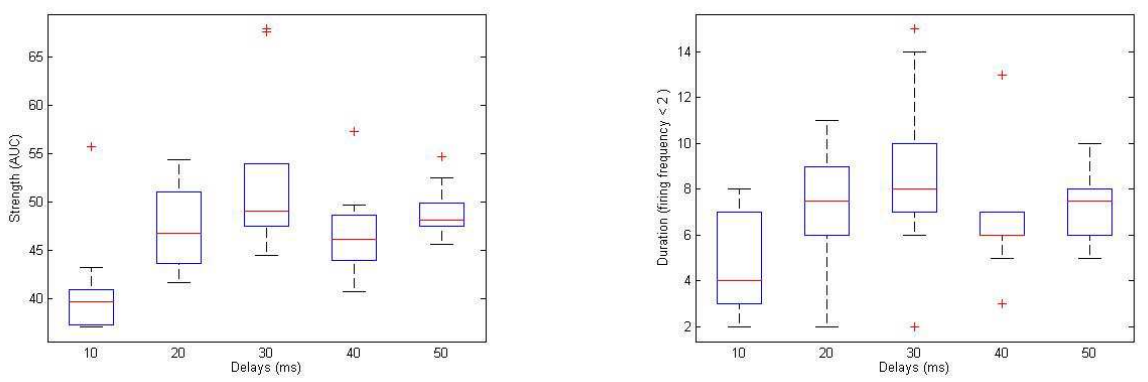

Fig. 4: Sustainability of polychronous groups when we vary the maximum length of the axonal conduction delays, or the time required for an action potential to travel from a pre- to a postsynaptic neuron. We get very small values for strength and duration for up to $10 \mathrm{~ms}$ delays, with very small variance especially for 10ms delays, and much bigger values for 20, 30, 40 and 50ms delays.

Another important parameter that affects network structure is the maximum delays between synaptic connections. Axonal conduction delays refer to the time required for an action potential to travel from its initiation site near the neuronal soma to the axon terminals, where synapses connect the soma with other neurons [17]. Evidence suggests that conduction time in the mammalian brain can reach from a few $\mathrm{ms}$ up to over $50 \mathrm{~ms}$ [20]. In the original model however, conduction delays had an upper limit of $20 \mathrm{~ms}$. In our experiment, we set the upper limit of axonal conduction delays to 50ms, to see again how it will affect sustainability of memory traces. This parameter can be interpreted in the brain as populations with short synapses, or areas with really high density, as mentioned above.

We get small values for strength and duration for up to $10 \mathrm{~ms}$ delays with very small variance, and increasingly much bigger values for 20, 30, 40 and 50ms delays (see Fig. 4). The big mean differences and the very small variance between our results indicate how crucial the maximum range of axonal conduction delays in the network is to PNG sustainability. This can also be indicated by the very small $p$ values after an ANOVA both for strength $(p=0.0006)$ and duration $(p=0.033)$. Axonal delays, influence the PNG locals, as it determines the "spread" of spike times locally. There may be a maximum of quality: If the spread is too narrow - no polygronous groups, because all need to fire more or less at once. If the spread is too big, the likelihood discreases of arrival of several spikes within the membrane time constant at a target neuron, so no PNGs either. 


\subsection{Small World Connectivity}
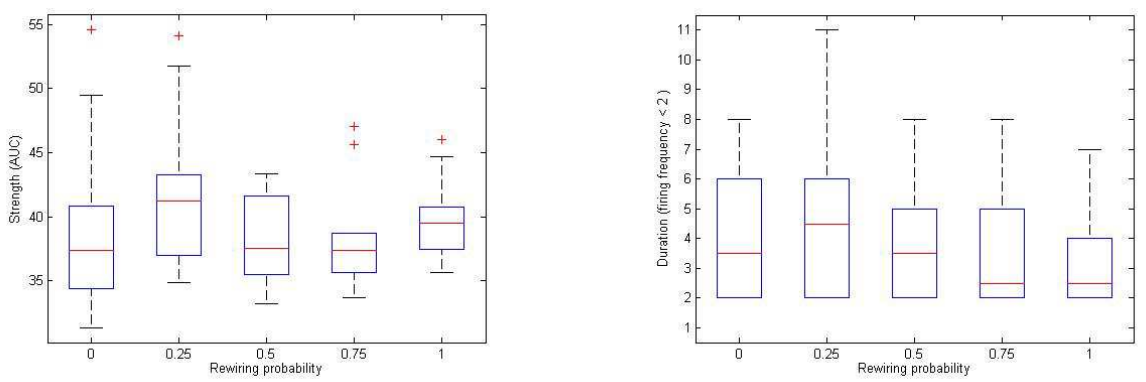

Fig. 5: Sustainability of polychronous groups when we vary the rewiring probability of a neighbouring synaptic connection to move from a local connectivity network $(b=0)$ to a random connectivity network $(b=1)$. There is an optimum mean value for $b=0.25$, however with a big variance in all results, especially in the sustainability duration (right).

For our next set of experiments we apply a small world connectivity pattern in the network [21]. Small-world has been widely used in recent years, both for its biological realism as well as delivering cost-effective information-processing systems $[22,23]$. We use a variation of the Watts-Strogatz algorithm [21] that produces graphs with small-world properties: We construct a regular lattice (here a neural network), a graph with $\mathrm{N}$ nodes (neurons) each connected to $\mathrm{K}$ neighbours, $\mathrm{K} / 2$ on each side. For every node $n_{i}=n_{0}, \ldots, n_{N-1}$ we take every edge (synapse) $n_{i}, n_{j}$ with $i<j$ and rewire it with probability $\beta$. Rewiring is done by replacing $\left(n_{i}, n_{j}\right)$ with $\left(n_{i}, n_{k}\right)$ where $k$ is chosen with uniform probability from all possible values. We vary the rewiring probability of a neighbouring synaptic connection to move from a local connectivity network $(\beta=0)$ to a random connectivity network $(\beta=1)$. In addition, we assign the delays according to the relevant distance between pre- and postsynaptic neurons.

There appears to be an optimum sustainability mean value for $\beta=0.25$ (see Fig. 5), however there is a big variance in all the results, especially in the sustainability duration (right) indicating that the differences between column means are not significant. This is also quantified after an ANOVA with which we get high $p$ values both for strength $(p=0.5432)$ and duration $(p=0.7811)$. Connectivity does not influence quality of PNG, as it does not matter for a group where the neurons that are part of as long as they have enough connections to the anchor neurons. So while the global connectivity looks different for a different network topology, the algorithm selects on those motifs that fit into the PNG pattern anyway, it explicitly affects a certain connectivity structure. These structures may be more or less likely to find in networks of different topologies - but once found, they behave and look the same as only the connections with a PNG are important, and those to the outside world.

\subsection{Excitation versus Inhibition}
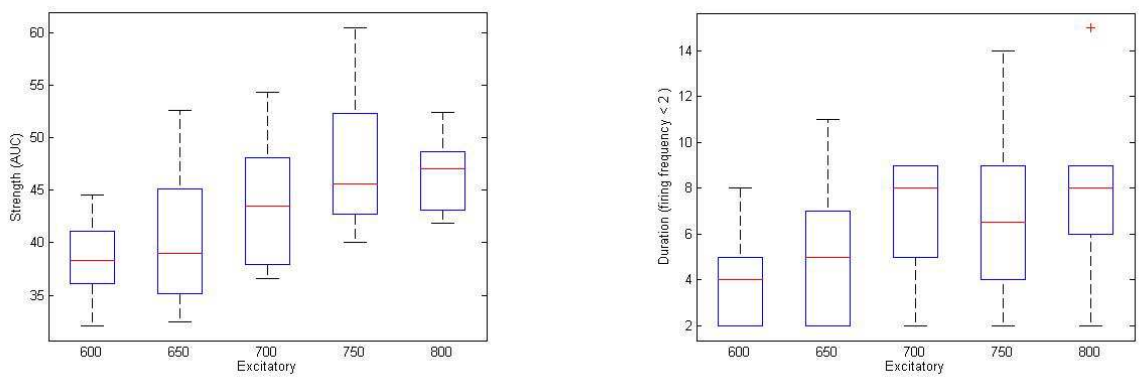

Fig. 6: Sustainability of polychronous groups when we vary the number of excitatory neurons in the model, keeping the total number of neurons at 1000. There is an increase to the strength and duration values as the number of excitatory neurons increase.

Cortical networks consist of two basic neural elements: excitatory principal cells and inhibitory interneurons. Research has suggested that in the cerebral cortex, excitatory synapses represent the majority 
of the synapses $(80 \%)$, however, there is evidence that areas such as the CA1 region of the hippocampus may have as many as 30,000 excitatory inputs and 1700 inhibitory inputs [17]. There are remarkable differences regarding the proportion, length, and density of excitatory and inhibitory synapses (asymmetric and symmetric synapses, respectively) between brain regions [17] as well as between mammals and their respective brain areas [19]. A detailed distribution of excitatory as well as inhibitory inputs on the somata and dendrites of neural cells, not only in working memory related brain areas, but also in the whole neural system remains elusive [17]. Thus, our line of focus is not to create a direct mapping of the excitatory versus inhibitory population ratios of specific brain areas, but to get a general view of how excitatory and inhibitory inputs affect the sustainability of PNGs. In this experiment we explore how sustainability strength and duration of polychronous groups is affected when we vary the number of excitatory neurons in the model, keeping the total number of neurons to 1000 .

There is a large increase to the strength mean and a moderate increase in duration (large variance for duration) mean as the number of excitatory neurons in the network increase (see Fig. 6), indicating that excitation is a crucial parameter for sustainability in the model. This can be quantified using ANOVA resulting to very small $p$ values for strength $(p=0.0022)$ and moderate for duration $(p=0.1284)$. The large number of inhibitory connections that a neuron receives can supress the synaptic dynamics of the constituent PNG neurons.

\section{Conclusion}

We explored how different parameters affect the sustainability of a polychronous group. The range of axonal conduction delays and excitatory neurons affect sustainability in the network, whereas network size, number of connections, connectivity pattern do not impact sustainability of a PNG. Previous work has shown that these parameters can affect memory content as quantity of emerging PNGs [12], however here they do not affect the intrinsic properties (quality) of PNGs due to the associative nature of the synaptic dynamics.

It appears that the short-term storage of information might not be that different between individuals or between mammals of differents brain sizes. Perhaps the small effect of these parameters might also give an explanation on the dissociation of intelligence and brain size between individuals. However, this might not be the case between species with enormous differences in brain scales, because on one hand specific parameters may not affect sustainability to a great extent, but on the other hand they affect the memory content in the form of the number of polychronous groups that emerge, i.e. the number of synaptic connections greatly affects the number of emerging polychronous groups [12]. Axonal delays and excitation results suggest that we need specific values of parameters as a percentage of the whole network in order to optimise sustainability. Here the bigger the number of excitatory neurons in the network the bigger sustainabilty follows. This could suggest that there is a bigger sustainability efficiency in brain areas where we have a big percentage of excitatory neurons i.e. in the hippocampus as opposed to smaller percentage in cortical areas [17]. This could also relate to the fMRI data results revealing that activation in left ventral lateral prefrontal cortex and temporoparietal junction predicted subsequent confidence ratings and in contrast, parahippocampal and hippocampal activity predicted the number of details remembered [13].

In addition to these fundamental structural differences, further detailed modelling of brain areas is necessary in order to predict the effect of sustainability not only in the context of short term memory, but its effect in other information processing mechanisms in the brain i.e. the role of the hippocampus to long term memory consolidation or spatial navigation [24]. This is in conjuction with the fact that spike-timing dependent plasticity has been uncovered in a variety of brain regions, including prefrontal, entorhinal, somatosensory and visual cortices, hippocampus, striatum, the cohlear nucleus and the amygdala, and in different mammals with different brain structures [16]. In future work, we will explore this sustainability to larger scale networks i.e. using massively parallel computing platforms, to better evaluate sustainability and elucidate the effect of parameters not shown here due to scale limitations. In addition, we will incorporate more biological details as they emerge as well as bring together modelling from different scales. For example we can integrate in our model realistic morphological models of dendrites [25] and explore different types of synaptic plasticity [26,27]. Furthermore, we will combine the macroscopic view of network modelling in the form of interconnected brain regions, and view the effect of sustainability on the neuronal oscillations and how that affects the propagation of the rhythms in association with in vivo brain imaging and electrophysiological observations. 


\section{References}

1. Fuster, J.M., Alexander, G.E., et al.: Neuron activity related to short-term memory. Science 173(3997) (1971) 652-654

2. Miyashita, Y.: Neuronal correlate of visual associative long-term memory in the primate temporal cortex. Nature 335(6193) (1988) 817-820

3. Funahashi, S., Bruce, C.J., Goldman-Rakic, P.S.: Mnemonic coding of visual space in the monkey's dorsolateral prefrontal cortex. Journal of Neurophysiology 61(2) (1989) 331-349

4. Zipser, D., Kehoe, B., Littlewort, G., Fuster, J.: A spiking network model of short-term active memory. The Journal of neuroscience 13(8) (1993) 3406-3420

5. Wang, X.J.: Synaptic basis of cortical persistent activity: the importance of nmda receptors to working memory. The Journal of Neuroscience 19(21) (1999) 9587-9603

6. Hempel, C.M., Hartman, K.H., Wang, X., Turrigiano, G.G., Nelson, S.B.: Multiple forms of short-term plasticity at excitatory synapses in rat medial prefrontal cortex. Journal of Neurophysiology 83(5) (2000) 3031-3041

7. Wang, X.J.: Synaptic reverberation underlying mnemonic persistent activity. Trends in neurosciences 24(8) (2001) 455-463

8. Machens, C.K., Romo, R., Brody, C.D.: Flexible control of mutual inhibition: a neural model of two-interval discrimination. Science 307(5712) (2005) 1121-1124

9. Mongillo, G., Barak, O., Tsodyks, M.: Synaptic theory of working memory. Science 319(5869) (2008) $1543-1546$

10. Sugase-Miyamoto, Y., Liu, Z., Wiener, M.C., Optican, L.M., Richmond, B.J.: Short-term memory trace in rapidly adapting synapses of inferior temporal cortex. PLoS computational biology 4(5) (2008) e1000073

11. Szatmáry, B., Izhikevich, E.M.: Spike-timing theory of working memory. PLoS computational biology 6(8) (2010) e1000879

12. Ioannou, P., Casey, M., Grüning, A.: Evaluating the effect of spiking network parameters on polychronization. In: Artificial Neural Networks and Machine Learning-ICANN 2012. Springer (2012) 255-263

13. Qin, S., van Marle, H.J., Hermans, E.J., Fernández, G.: Subjective sense of memory strength and the objective amount of information accurately remembered are related to distinct neural correlates at encoding. The Journal of Neuroscience 31(24) (2011) 8920-8927

14. Izhikevich, E.M.: Polychronization: computation with spikes. Neural computation 18(2) (2006) 245-282

15. Izhikevich, E.M., et al.: Simple model of spiking neurons. IEEE Transactions on neural networks 14(6) (2003) 1569-1572

16. Sjöström, J., Gerstner, W.: Spike-timing dependent plasticity. Scholarpedia 5(2) (2010) 1362

17. Shepherd, G.M.: The Organization of the Brain. Oxford University Press (2004)

18. Braver, T.S., Cole, M.W., Yarkoni, T.: Vive les differences! individual variation in neural mechanisms of executive control. Current opinion in neurobiology 20(2) (2010) 242-250

19. DeFelipe, J.: The evolution of the brain, the human nature of cortical circuits, and intellectual creativity. Frontiers in neuroanatomy 5 (2010) 29-29

20. Swadlow, H.A., Waxman, S.G.: Axonal conduction delays. Scholarpedia 7(6) (2012) 1451

21. Watts, D.J., Strogatz, S.H.: Collective dynamics of 'small-world'networks. Nature 393(6684) (1998) 440-442

22. Bassett, D.S., Bullmore, E.: Small-world brain networks. The neuroscientist 12(6) (2006) 512-523

23. Vertes, P.E., Duke, T.: Effect of network topology on neuronal encoding based on spatiotemporal patterns of spikes. HFSP journal 4(3-4) (2010) 153-163

24. Buzsaki, G.: Hippocampus. Scholarpedia 6(1) (2011) 1468

25. Cuntz, H., Forstner, F., Borst, A., Häusser, M.: One rule to grow them all: A general theory of neuronal branching and its practical application. PLoS Comput Biol 6(8) (08 2010) e1000877

26. Notley, S., Gruning, A.: Improved spike-timed mappings using a tri-phasic spike timing-dependent plasticity rule. In: Neural Networks (IJCNN), The 2012 International Joint Conference on. (June 2012) 1-6

27. Chrol-Cannon, J., Grüning, A., Jin, Y.: The emergence of polychronous groups under varying input patterns, plasticity rules and network connectivities. In: Proceedings of the International Joint Conference on Neural Networks (IJCNN). (2012) 